\title{
Amyloglucosidase Catalyzed Syntheses of Bakuchiol Glycosides in Supercritical Carbon Dioxide
}

\author{
Balaraman Manohar, Soundar Divakar, ${ }^{\star}$ and Kadimi Udaya Sankar \\ Food Engineering Department, Central Food Technological Research Institute, Avsore 570020, India \\ E-mail: manoharmlsorea à woo co in or manoharbiácfnires in \\ Fermentation Technology and Bioengineering Department, Central Food Technological Research Institute, \\ Mrisore 570020. India \\ Received January 31, 2009, Accepted June 23, 2009
}

\begin{abstract}
Enzymatic syntheses of water soluble Bakuchiol glycosides were carried out in di-isopropyl ether organic media using amy loglucosidase from Rhizopus mold. The reactions were carried out under conventional reflux conditions and in supercritical $\mathrm{CO}_{2}$ atmospheric conditions. Out of the eleven carbohydrate molecules employed for the reaction, D-glucose, D-ribose and D-arabinose gave glycosides in yields of $9.0 \%$ to $51.4 \%$ under conventional reflux conditions. Under supercritical $\mathrm{CO}_{2}$ atmosphere ( 100 bar pressure at $50^{\circ} \mathrm{C}$ ), bakuchiol formed glycosides with Dglucose, D-galactose, D-mannose, D-fructose, D-ribose, D-arabinose, D-sorbitol and D-mannitol in y ields ranging from $9 \%$ to $46.6 \%$. Out of the bakuchiol glycosides prepared, $6-O-(6$-D-fructofruranosyl)bakuchiol showed the best antioxidant ( $1.4 \mathrm{mM})$ and $\mathrm{ACE}$ inhibitory activities $(0.64 \mathrm{mM})$
\end{abstract}

Key Wonds: ACE inhibition. Antioxidant activity. Bakuchiol, Bakuchiol glycosides. Glycosylation

\section{Introduction}

Bakuchiol (I) is a biologically active mono-terphenic phenolic compound having a single hydroxyl group on the aromatic ring and an unsaturated hỵdrocarbon chain. Bakuchiol is isolated from the seeds of Chiba Psoralea corvlifolia $\mathrm{L}$. distributed all over the subcontinent extending well into Southeast Asia. The seed-oil is used externally for the treatment of leucoderma. psoriasis and leprosy in Indian folk medicine. The plant, known as Bakuchi in Sanskrit. has been used in Ayurvedic medicinal system as a cardiac tonic, vasodilator and pigmentor. It is widely used in Chinese medicine to treat a variety of diseases and possesses antitumor. antibacterial. cytotoxic and antihelmenthic properties. ${ }^{2}$ Thermally sensitive bakuchiol, psoralen and isosporalen, the major components present in the seed possess important therapeutic properties.

Earlier studies on the principal components of Chiba seed have shown significant antibacterial and antioxidant activities and a great potential for use in food additives and mouthwash for preventing and treating dental caries. ${ }^{3.4}$ Synthetic antioxidants such as butylated hy'droxyl-anisole (BHA) widely used in food industries are known to cause liver damage and carcinogenesis. ${ }^{5,6}$ Hence, development of effective non-toxic antioxidants from natural sources is very much desired.

Bakuchiol exhibits poor water solubility, stability and absorbability. Glycosylation improves the pharmacological property by increasing the water solubility of Bakuchiol. One-step enzymatic glycosylation is useful for the preparation of glycosides rather than chemical glycoșy lation, which requires large number of protection-deprotection steps

Production of fine chemicals results in generation of considerable solvent waste as synthesis generally includes number of steps. Typically one $\mathrm{kg}$ of end product leads to generation of $15 \mathrm{~kg}$ of wastes. Most of them are solvents and by-products.
Therefore. several reactions were performed in water or in supercritical fluids (SCF) recently. Manufacturing processes in liquid and supercritical fluids (SCF) are advantageous in terms of energy reduction, ease of product recoven. lesser cost of downstream processing and reduction in side reactions.

The advantage of using supercritical carbon dioxide as a reaction medium is well documented. ${ }^{*-1 i j}$ Recently. use of supercritical $\mathrm{CO}_{2}\left(\mathrm{SCCO}_{2}\right)$ as a solvent in enzyme-catalysed reactions has been a matter of considerable research interest because of its favorable transport properties that can accelerate masstransfer-limited enzymatic reactions. Since the first reports on the use of SCF as reaction media in 1980 s. several studies on enzymatic oxidation. hydrolysis, transesterification, esterification. interesterification and enantioselective synthesis have proven the feasibility of enzymatic reactions in supercritical fluids. ${ }^{[1.1 ?}$ The temperature range employed in supercritical carbon dioxide processing is favorable for the use of enz'mes as catalysts besides providing a medium for the recovery of products or reactants without many unwanted process steps.

Enzymatic glycosylation of bakuchiol with different carbohydrates in supercritical fluid media has not been reported in literature. Hence. the present study is attented to prepare water soluble bakuchiol glycosides enzymatically using anyloglucosidase from Rhizopus mold utilizing different carbohydrate nolecules in two different environments: one under conventional reflux conditions and another under $\mathrm{SCCO}_{2}$ conditions.

\section{Experimental Section}

Enzymes. Anyloglucosidase from Rhizopus sp. was purchased from Sigma Comparyy. St. Louis. MO. USA. Anny loglucosidase activity ${ }^{18}$ was found to be 11.2 activity units (AU- $\mu \mathrm{mol} /$ (mg. enzyme. min)).

Chemicals and reagents. D-Galactose and D-fructose were 
<smiles>C=CC(C)(CC=Cc1ccc(O)cc1)CCC=C(C)C</smiles>

Bakuchiol

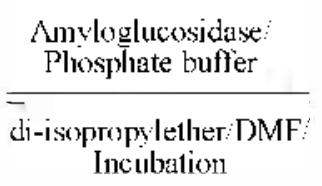<smiles>C=CC(C)(C=Cc1ccc(OC)cc1)CCCC(C)C</smiles>

Bakuchiol glycosides

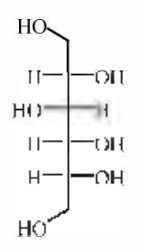

D-Sorbitol

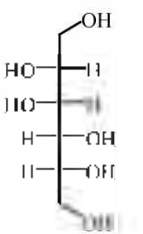

D-Mannitol

Scheme 1. Syntheses of Bakuchiol glycosides

procured from HiMedia Pvt. I.td, India. I)-(jlucose and sucrose purchased trom SD Fine Chemicals (India) Ltd., D-mannose, D-arabinose, D-ribose, D-sorbitol and D-mannitol, from Loba Chemie Pvt. I.td., India and maltose trom Sigma Chemical Co., St. Louis, MO, USA were employed. Lactose, HI'LC' grade acetonitrile and di-isopropyl ether were from Sisco Research Laboratories P'vt. Ltd., India. Li-isopropyl ether was distilled once betore use.

Glycosylation procedure-conventional neflux method. Syntheses of bakuchiol glycosides involved refluxing bakuchiol (0.5 mmol) with 1.0 mmol carbohydrates in $100 \mathrm{mI}$, di-isopropyl ether in presence of anyloglucosidase $(40 \% \mathrm{w} / \mathrm{w}$ carbohydrates), l)MF $5.0 \mathrm{mI}$ and $0.1 \mathrm{mM}$ (in $100 \mathrm{mI}$, di-isopropv1 ether), $\mathrm{pH} 6.0$ buffer for an incubation period of $72 \mathrm{~h}$ at $68^{\circ} \mathrm{C}$ (Scheme 1). Alter the reaction, the solvent was evaporated and the enzyme denatured at $100^{\circ} \mathrm{C}$ by holding in a boiling water bath for $5 \sim 10 \mathrm{~min}$. The residue containing unreacted bakuchiol carbohydrates, along with the product glycosides were dissolved in $15 \sim 20 \mathrm{~mL}$ of water and the reaction misture extracted with hexane to remove unreacted bakuchiol. The dried residue was subjected to HPLC analysis to delemine the extent of conversion. All the reactions were perloned in triplicate and the mean values are shown in tables. Unteacted

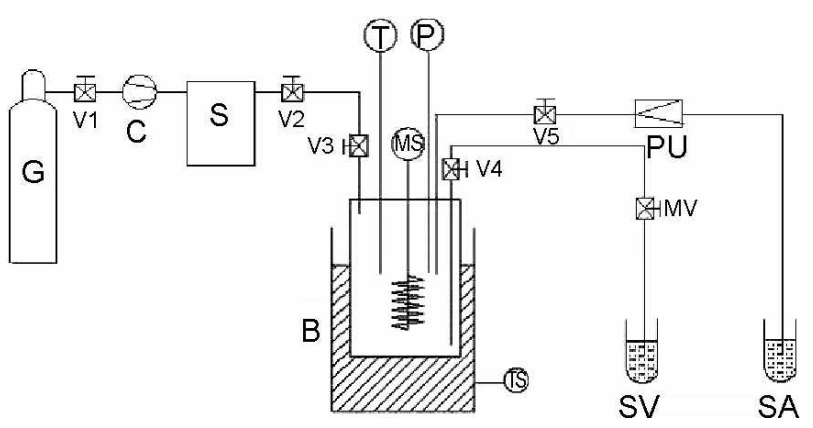

Figure 1. Process schematic diagram of experimental set up to carry out the reactions in $\mathrm{SCF} \mathrm{CO}$. G. Gas cylinder, C. Compressor. $\mathrm{S}$. Surge tank; B. Berghof autoclave: 'l, lemperature indicator: P, Pressure indicator: MS Magnetic stirrer; V1-V5, High pressure needle valve: MV. Micrometer valve; SV. Sampling valve: SA. Saline solution: TS. 'Thermostat: PU. Pump. carhohydrate was separated from the product glycosides by size exclusion chromatography using Sephadex G 15 column $(100 \mathrm{~cm} \times 1 \mathrm{~cm})$, eluting with water at $1 \mathrm{~mL} / \mathrm{h}$ rate. Individual glycosides could not be separated satisfactorily, due to similar polarity of the glycosides fommed.

Syntheses of the other bakuchiol glycosides were carried out at the above determined conditions, with bakuchiol and carbohydrates: aldohexoses - D-glucose, D-galactose and D-mannose; ketohexose - D-lructose; pentoses - D-ribose and D-arabinose; disaccharides - maltose, lactose and sucrose: sugar alcohol - I)-sorbitol and I)-mannitol. The conditions employed with the enzymes are: bakuchiol $(0.5 \mathrm{mmol})$ and carhohydrate ( $1.0 \mathrm{mmol}$ ), any loolucosidase $(40 \% \mathrm{w} / \mathrm{w}$ carbohydrates), $0.2 \mathrm{mM}, \mathrm{pH} 6.0$ phosphate buller and 72 h of incubation period.

Gycosylation procedure at $\mathbf{S C C O}_{2}$ conditions. Syththeses of the bakuchiol glycosides were carried out with bakuchiol and carbohydrates in supercritical $\mathrm{CO}_{2}$ atmosphere of $100 \mathrm{bar}$ pressure at 50 " $\mathrm{C}$. The reactor vessel along with the $\mathrm{CO} \mathrm{O}_{2}$ supply system is shown schematically in Figure l. It consists of a reactor of $120 \mathrm{~mL}$ capacity with a magnetic stirrer and a recirculating lluid loop by means ol a pressure dillerential for sampling through a Rlicodyne valve with $0.5 \mathrm{mI}$, loop for sampling. Total volume of about $50 \mathrm{~mL}$ of the reactor vessel was themostatically controlled to maintain a constant temperature. Reaction Process conditions employed are: bakuchiol $(0.5$ munol) and carbohydrate ( $1.0 \mathrm{mmol})$, any loglucosidase ( $40 \%$ w/w carbohydrates). DMT $15 \mathrm{mI}$, and $0.1 \mathrm{mM}, \mathrm{pH}$ 6.0 phosphate buffer and $24 \mathrm{~h}$ of incubation period. The $\mathrm{CO}_{2}$ was then released and the reaction products were taken out in $15-20$ $\mathrm{mL}$ of water, evaporated to dryness and subjected to analy ses by HI'LC' and NMR.

Antioxidant activity measurement. Antioxidant activily of bakuchiol and bakuchiol glycosides were dekmined by DPPI (2,2 diphenyl-1-picryl hydrazyl) radical scavenging method. ${ }^{1 !}$ The reaction miature contained $0.1 \mathrm{~mL}$ of test sample $(5 \sim 10 \mathrm{mM})$ and $1.0 \mathrm{~mL}$ of $\mathrm{J}) \mathrm{P}^{\mathrm{P}} \mathrm{l}^{\mathrm{H}} \mathrm{H}(0.36 \mathrm{mM})$ with the final volume adjusted $102.0 \mathrm{~mL}$ of $0.1 \mathrm{M}$ Iris $\mathrm{HCl}$ buffer $(\mathrm{pH} 7.4$ ). The reaction mixture was incubated at room temperature for 20 minutes in the dark and the antioxidant activity was detcrunined by monitoring 
the decrease in absorbance at $517 \mathrm{~nm}$ on an UV-Visible spectrophotometer (Shimadzu. UV 1601). Butylated hydroxy anisole (BHA- $5.6 \mathrm{mM}$ ) was used as the positive control. IC $\mathrm{S}_{10}$ value for the antioxidant activity was expressed as the concentration of the glycoside corresponding to $50 \%$ decrease in absorbance value of DPPH from a plot of decrease in absorbance versus concentration of the glycoside. Error in activity measurements is $\pm \mathbf{5 \%}$.

Angiotensin Converting Enzyme (ACE) inhibition assay. $\mathrm{ACE}$ inhibition assay for the bakuchiol and bakuchiol glycosides were performed with ACE isolated from pig lung by the Cushman and Cheung method. Aliquots of glycoside solutions in the concentration range 0.2 to $1.8 \mathrm{mM}(0.1 \mathrm{~mL}$ to 0.8 $\mathrm{mL}$ of $2.0 \mathrm{mM}$ stock solution) were taken and to this $0.1 \mathrm{~mL}$ of $\mathrm{ACE}$ solution $(0.1 \%$ in $0.1 \mathrm{M}$ phosphate buffer $\mathrm{pH} 8.3$ containing $300 \mathrm{mM} \mathrm{NaCl}$ ) along with $0.1 \mathrm{~mL}$ of $2.5 \mathrm{mM}$ hippurylL-histidyl-L-leucine (HHL) were added and incubated in a water bath for $30 \mathrm{~min}$ at $37^{\circ} \mathrm{C}$. Blanks were performed without the enzyme. Hippuric acid released was estimated from a calibration plot yielding $0.0105 \mathrm{Abs}$ units/nmol hippuric acid. Percentage inhibition was expressed as the ratio of specific activity of ACE in presence of the inhibitor to that in its absence. the latter being considered as $100 \%$. $\mathrm{IC}_{5 i}$ value was expressed as the concentration of the inhibitor required for $50 \%$ reduction in ACE specific activity. Error in measurements is $\pm \mathbf{5 \%}$.

${ }^{1} \mathrm{H}$ and ${ }^{13} \mathrm{C}$ nuclear magnetic resonance. Two-dimensional Heteronuclear Single Quantum Coherence Transfer spectra (2D HSQCT) were recorded on a Brüker DRX-500 MHz spectrometer operating at $500.13 \mathrm{MHz}$ for ${ }^{1} \mathrm{H}$ and $125 \mathrm{MHz}$ for ${ }^{13} \mathrm{C}$ at $35^{\circ} \mathrm{C}$. Proton and carbon $90^{\circ}$ pulse widths were 12.25 and $10.5 \mu$. respectively. Chemical shifts were expressed in ppm relative to internal tetramethylsilane standard. About $40 \mathrm{mg}$ of the glycoside simple dissolved in DMSO- $d_{6}$ was used for recording the spectra in magnitude mode with sinusoidal-shaped $z$-gradients of strength 25.7 .15 .42 and $20.56 \mathrm{G} / \mathrm{cm}$ with a gradient recovery delay of $100 \mu$ s to defocus unwanted coherences. Increment of $t_{1}$ was in 256 steps with a computer memory size of $+\mathrm{kB}$. The spectra were processed using unshifted and $\pi / 4$ shifted sine bell window function in $F_{1}$ and $F_{2}$ dimensions. respectively

Product characterization. Isolated glycosides besides measuring melting point and optical rotation were also characterized by recording UV, IR. Mass and 2D-HSQCT NMR spectra which provided good information about the nature and type of products. In 2D-HSQCT some of the assignments are interchangeable. Only resolvable signals are shown. The glycosides. being surfactant molecules tend to aggregate in solution giving rise to broad signals. thus making it difficult to resolve the coupling constant values of some of the signals.

Spectral characterization. Bakuchiol: Solid. UV $\left(\hat{t}_{\text {max }}\right)$ : $226.5 \mathrm{~nm}\left(\sigma \rightarrow \sigma^{*}, \varepsilon_{\geq 36.5}-3+81 \mathrm{M}^{-1}\right), 299.0 \mathrm{~nm}\left(\mathrm{n} \rightarrow \pi^{*} . \varepsilon_{195.5}\right.$ $\left.-896 \mathrm{M}^{-1}\right)$; $\mathbb{R}\left(\mathrm{KBr}\right.$. stretching frequency. $\left.\mathrm{cm}^{-1}\right): 3320(\mathrm{OH})$. $1373(\mathrm{C}=\mathrm{C}) .2928(\mathrm{CH}): 2 \mathrm{D}-\mathrm{HSQCT}\left(\mathrm{DMSO}-d_{6}\right){ }^{\mathrm{j}} \mathrm{H}$ NMR $\hat{\delta}_{\mathrm{ppm}}$ (500.13): $7.27(\mathrm{H}-2) .7 .19(\mathrm{H}-3) .6 .69(\mathrm{H}-4), 6.71(\mathrm{H}-5), 6.04$ (H-7). $6.17(\mathrm{H}-8) .1043$ (H-10a). 1.21 (H-10b). $1.95(\mathrm{H}-11) .4 .66$ (H-12), 1.62 (H-14). 1.52 (H-15). 5.9 (H-16), 1.14 (H-17). 5.08 (H-18a), $5.02(\mathrm{H}-18 \mathrm{~b}):{ }^{13} \mathrm{C} \mathrm{NMR} \delta_{p \mathrm{pmon}}(125 \mathrm{MHz}): 126.6(\mathrm{Cl})$, 127.2 (C2) 127.2 (C3), $115.4(\mathrm{C}+) .115 .4$ (C5). 156.1 (C6).
128.4 (C7). 134.1 (C8), 42.2 (C9), 41.0 (C10). 25.5 (C11), 124.2 (Cl2), 130.6 (Cl3). 23.1 (Cl4). 22.9 (Cl5). 146.0 (Cl6). $17.5(\mathrm{Cl}) .111 .8(\mathrm{Cl})$.

6-O-(D-Glucopyranosyl)balachiol: Solid. UV $\left(\lambda_{\text {max }}\right): 191.5$ $\operatorname{nm}\left(\sigma \rightarrow \sigma^{*}, \varepsilon_{191.5}-3074 \mathrm{M}^{-1}\right) .229 .5 \mathrm{~nm}\left(\sigma \rightarrow \sigma^{*}, \varepsilon_{229.5}-1774\right.$ $\left.\mathrm{M}^{\mathrm{l}}\right), 275.5 \mathrm{~nm}\left(\pi \rightarrow \pi^{*}, \varepsilon_{2}: 511 \mathrm{M}^{-1}\right)$; IR (KBr. stretching frequency. $\left.\mathrm{cm}^{-1}\right): 3371(\mathrm{OH}), 1381$ (glycosidic aryl alkyl $\mathrm{C}-\mathrm{O}-\mathrm{C}$ asynmetrical), 1080 (glycosidic aryl alkyl C-O-C symmetrical), $1380(\mathrm{C}=\mathrm{C}) .2937(\mathrm{CH})$; MS (n/z) $+19[\mathrm{M}+1]^{+}$. 2D-HSQCT (DMSO-d $\left.d_{6}\right)$ C1(a-glucoside: ${ }^{1} \mathrm{H}$ NMR $\delta_{\mathrm{ppm}}(500.13) \mathrm{Glu}: 47$ (H-1 $\alpha$. d. $J=2.7 \mathrm{~Hz}), 3.57(\mathrm{H}-3 \alpha), 3.11(\mathrm{H}-4 \alpha)$ : Bakuchiol: $7.26(\mathrm{H}-3) .2 .01(\mathrm{H}-\mathrm{ll}) .4 .9(\mathrm{H}-12) .0 .93(\mathrm{H}-17) .{ }^{13} \mathrm{C} \mathrm{NMR} \delta_{\mathrm{pp} \pi \mathrm{r}}$ (125 MHz): Glu: $95.0(\mathrm{Cl} \alpha) .75 .5(\mathrm{C} 3 \alpha), 70.8(\mathrm{C}+\alpha), 63.3$

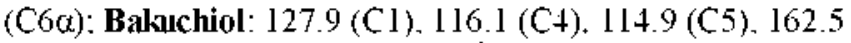

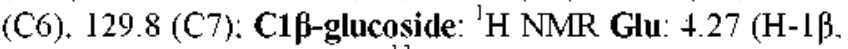
d. $J=6.7 \mathrm{~Hz}), 3.67(\mathrm{H}-6 \mathrm{a}),{ }^{12} \mathrm{C}$ NMR $\delta_{\text {igm }}$ Glu: $101.8(\mathrm{Cl} \beta)$, $75.5(\mathrm{C} 2 \beta) .76 .3(\mathrm{C} 3 \beta)$.

6- $O$-(D-Galactopyranosyl)bakuchiol: Solid, UV $\left(\lambda_{\text {this }}\right)$ : $191.5 \mathrm{~nm}\left(\sigma \rightarrow \sigma^{*} . \varepsilon_{191.5}-2983 \mathrm{M}^{-1}\right) .231 .5 \mathrm{~nm}\left(\sigma \rightarrow \sigma^{*}, \varepsilon_{231.5}\right.$ $\left.-1123 \mathrm{M}^{-1}\right), 274.5 \mathrm{lum}\left(\pi \rightarrow \pi^{*} . \varepsilon_{274.5}-257 \mathrm{M}^{-1}\right)$ : IR (KBr. stretching frequency, $\left.\mathrm{cm}^{-1}\right): 3319(\mathrm{OH}) .12+6$ (glycosidic aryl alkyl C-O-C asymmetrical), 1064 (glycosidic aryl alkyl C-O-C șmmetrical). $1361(\mathrm{C}=\mathrm{C}) .2917 .6(\mathrm{CH})$; $\mathrm{MS}(\mathrm{m} / \mathrm{z})+19[\mathrm{M}+1]^{+} .2 \mathrm{D}$ HSQCT (DMSO- $\left.d_{6}\right)$ C1 to-galactoside: ${ }^{1} \mathrm{H} N M R \delta_{\mathrm{FFm}}(500.13)$

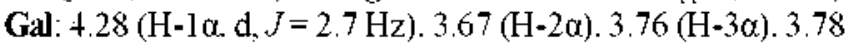
$(\mathrm{H}-4 \alpha) .3 .66(\mathrm{H}-5 \alpha)$ : Bakuchiol: $7.1+(\mathrm{H}-2) .7 .16(\mathrm{H}-3) .6 .50$ $(\mathrm{H}-4) .6 .52(\mathrm{H}-5), 6.13(\mathrm{H}-8), 1.14(\mathrm{H}-10), 1.13(\mathrm{H}-17):{ }^{13} \mathrm{C}$ NMR $\delta_{\text {pjin }}(125 \mathrm{MHz})$ : Gal: $95.4(\mathrm{Cl} \alpha), 68.4(\mathrm{C} 2 \alpha) .74 .7(\mathrm{C}+\alpha)$, 62.7 (C6 $\alpha$ ): Bakuchiol: 130.6 (C3). 162.5 (C6). 15.6 (Cl7): C1 p-galactoside: ${ }^{1} \mathrm{H}$ NMR Gal: $4.63(\mathrm{H}-1 \alpha, \mathrm{d}, J=3.4 \mathrm{~Hz}) .3 .29$ $(\mathrm{H}-\mathrm{I} \beta, \mathrm{d}, J=7.2 \mathrm{~Hz}) .3 .33(\mathrm{H}-5 \beta):{ }^{13} \mathrm{C}$ NMR $\delta_{\mathrm{pF}}$ Gal: 101.8

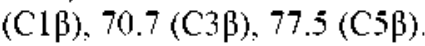

6-O-(D-Manuopyranosyl)bakuchiol: Solid, UV $\left(\lambda_{\text {miks }}\right): 191.5$ $\operatorname{nm}\left(\sigma \rightarrow \sigma^{*}, \varepsilon_{191.5}-2353 \mathrm{M}^{-1}\right) .225 .5 \mathrm{~nm}\left(\sigma \rightarrow \sigma^{*} . \varepsilon_{225.5}-120\right.$ $\left.\mathrm{M}^{-1}\right), 275.0 \mathrm{lml}\left(\pi \rightarrow \pi^{*}, \varepsilon_{2},-47 \mathrm{M}^{-1}\right)$ : IR (KBr. stretching frequency. $\left.\mathrm{cm}^{-1}\right): 2925(\mathrm{OH}), 1241$ (glycosidic anyl alkyl $\mathrm{C}-\mathrm{O}-\mathrm{C}$ asymmetrical), 1059 (glycosidic aryl alkyl C-O-C symunetrical). $1440(\mathrm{C}=\mathrm{C}) .2925(\mathrm{CH}): \mathrm{MS}(\mathrm{m} / \mathrm{z}) 4 \mathrm{i} 9[\mathrm{M}+\mathrm{I}]^{+} .2 \mathrm{D}-\mathrm{HSQCT}$ (DMSO- $d_{6}$ ) C1 $t$-Mannoside: ${ }^{1} \mathrm{H}$ NMR $\delta_{\mathrm{ppm}}(500.13)$ Man: 4.95 $(\mathrm{H}-1 \alpha . \mathrm{d}, J=1.6 \mathrm{~Hz}), 3.29(\mathrm{H}-3 \alpha) .3 .02(\mathrm{H}-5 \alpha), 3.68(\mathrm{H}-6 \alpha)$; Baluchiol: 1.46 (H-10a). 1.47 (H-10b), $0.922(\mathrm{H}-17):{ }^{13} \mathrm{C}$ NMR $\delta_{\text {jpon }}(125 \mathrm{MHz}$ ): Man: $94.76(\mathrm{Cl} \alpha)$ : Balachiol: 132.1 (C13), $14.3(\mathrm{Cl} 7)$.

6-O-(D-Fuctofuranosyl)balachiol: Solid, UV $\left(\lambda_{\max }\right): 191.5$ $\operatorname{nnt}\left(\sigma \rightarrow \sigma^{*}, \varepsilon_{19] 5}-206+\mathrm{M}^{\mathrm{l}}\right), 229.5 \mathrm{nun}\left(\sigma \rightarrow \sigma^{*} . \varepsilon_{25}-603\right.$ $\left.\mathrm{M}^{1}\right)$. $\left(\mathrm{n} \rightarrow \pi^{*} . \varepsilon_{256.5}-239 \mathrm{M}^{-1}\right) .288 .5 \mathrm{~mm}\left(\pi \rightarrow \pi^{*} . \varepsilon_{288.5}-222\right.$ $\mathrm{M}^{\mathrm{l}}$ ); IR ( $\mathrm{KBr}$, stretching frequency. $\mathrm{cm}^{.1}$ ): $3382.8(\mathrm{OH}) .1244$ (glycosidic añl alkyl C-O-C așymmetrical). 1060 (gly cosidic aryl alkyl C-O-C symmetrical), $1+16(\mathrm{C}=\mathrm{C}), 2930.1(\mathrm{CH})$; $\mathrm{MS}$ $(\mathrm{m} / \mathrm{z}) 419[\mathrm{M}+\mathrm{l}]^{+}$. 2D-HSQCT (DMSO- $\left.d_{6}\right)$ C6-fuctoside: ${ }^{\mathrm{H}}$ NMR $\delta_{\mathrm{ppm}}(500.13)$ Fur: $3.53(\mathrm{H}-3), 3.69(\mathrm{H}-4), 3.57(\mathrm{H}-5)$. 3.49 (H-6): Bakuchiol: 1.42(H-10a). 1.43 (H-10b), 1.13 (H-17): ${ }^{13} \mathrm{C}$ NMR $\dot{\partial}_{\mathrm{ppm}}(125 \mathrm{MHz})$ : Fu: 104.2 (C2). 70.7 (C3). 72.6 (C4). 71.6 (C5), 63.4 (C6): Balauchiol: 35.9 (C9), 38.2 (C10), 27.1 (Cl1). $14.0(\mathrm{Cl} 7)$.

6-O-(D-Ribofuranosyl)bakuchiol: Solid, UV $\left(\lambda_{\max }\right): 191.5$ $\operatorname{nnl}\left(\sigma \rightarrow \sigma^{*} . \varepsilon_{1915}-5015 \mathrm{M}^{\mathrm{l}}\right), 222.5 \mathrm{lnm}\left(\sigma \rightarrow \sigma^{*}, \varepsilon_{25}-2146\right.$ $\mathrm{M}^{-1}$ ). $260.4 \mathrm{~nm}\left(\pi \rightarrow \pi^{*}, \varepsilon_{2} 60.4-971 \mathrm{M}^{-1}\right)$ : IR (KBr. stretching 
frequency, $\left.\mathrm{cm}^{-1}\right): 3350(\mathrm{OH}) .1241$ (glycosidic aryl alkyl C-O-C asymmetrical). 1085 (glycosidic aryl alkyl C-O-C stmmetrical). $1416(\mathrm{C}=\mathrm{C}) .2930(\mathrm{CH}): \mathrm{MS}(\mathrm{m} / \mathrm{z}) 388[\mathrm{M}]^{-} .2 \mathrm{D}-\mathrm{HSQCT}$ (DMSO- $d_{b}$ ) Cla-riboside: 'H NMR $\delta_{\text {ppen }}(500.13)$ Rib: 4.64

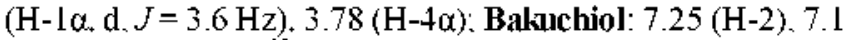

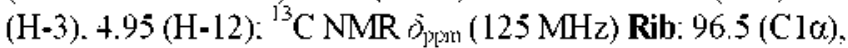
71.1 (C2 $\alpha$ ): Bakuchiol: 127.8 (C2), 130.1 (C7), 26.9 (C11):

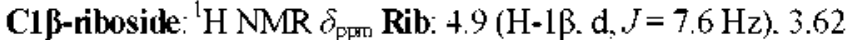

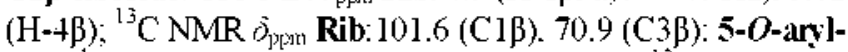
ated: ${ }^{1} \mathrm{H}$ NMR Rib: $3.55(\mathrm{H}-\mathrm{l} \alpha$ d. $J=2.9 \mathrm{~Hz}):{ }^{13} \mathrm{C}$ NMR $\delta_{\mathrm{ppr}}$ Rib: 62.1 (C5 $\alpha)$.

6-O-(D-Aalbinofuranosyl)baluchiol: Solid UV (hax $): 191.5$ $\operatorname{run}\left(\sigma \rightarrow \sigma^{*}, \varepsilon_{191}-6351 \mathrm{M}^{\mathrm{j}}\right) \cdot 221.4 \operatorname{sun}\left(\sigma \rightarrow \sigma^{*}, \varepsilon_{* \pm 1}-2701\right.$ $\left.\mathrm{M}^{-\mathrm{l}}\right) .259 .5 \mathrm{~nm}\left(\pi \rightarrow \pi^{*}, \varepsilon_{2595}-1241 \mathrm{M}^{\mathrm{l}}\right)$ : IR (KBr. stretching frequency $\left.\mathrm{cm}^{-1}\right): 3302(\mathrm{OH}) \cdot 1240$ (glycosidic aryl alkyl C-O-C asymunetrical). 1085 (glycosidic aryl alkyl C-O-C symmetrical), $140+(\mathrm{C}=\mathrm{C}) .2926(\mathrm{CH}): \mathrm{MS}(\mathrm{m} / \mathrm{z}) 427[\mathrm{M}+\mathrm{K}]^{+} .2 \mathrm{D}-\mathrm{HSQCT}$ (DMSO- $\left.d_{6}\right)$ Cla-arabinoside: ${ }^{l} \mathrm{H}$ NMR $\delta_{10 \mathrm{~m}}(500.13)$ Ara: 5.00 (H-la. d $J=3.4 \mathrm{~Hz}) .3 .70(\mathrm{H}-4 \alpha) .3 .52(\mathrm{H}-2 \alpha)$. Balauchiol: $6.34(\mathrm{H}-8), 0.94(\mathrm{H}-17) .5 .09(\mathrm{H}-18 \mathrm{a}), 5.32(\mathrm{H}-18 \mathrm{~b}):{ }^{13} \mathrm{C} \mathrm{NMR}$ $\delta_{\mathrm{p} p \mathrm{~m}}(125 \mathrm{MHz})$ : Ara: $95.9(\mathrm{Cl} \alpha), 75.5(\mathrm{C} 2 \alpha)$; Baluchiol: 116.1 (C4) 127.90 (C2) 28.68 (C 1 ) $: \mathrm{C} 1 \beta$-arabinoside: ${ }^{1} \mathrm{H}$ NMR oprm Ara: $4.96(\mathrm{H}-1 \beta . \mathrm{d}, J=6.2 \mathrm{~Hz}), 3.40(\mathrm{H}-4 \beta):{ }^{13} \mathrm{C}$

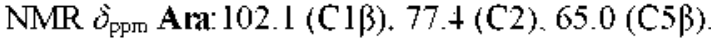

6-O-(1-D-Sonbitol)balauchiol: Solid. UV $\left(\lambda_{\text {max }}\right): 191.0 \mathrm{~nm}$ $\left(\sigma \rightarrow \sigma^{*} . \varepsilon_{191}-2092 \mathrm{M}^{\mathrm{l}}\right) .226 .0 \mathrm{~nm}\left(\sigma \rightarrow \sigma^{*} . \varepsilon_{2 z \sigma 0}-446 \mathrm{M}^{\mathrm{l}}\right)$. $255.0 \mathrm{~nm}\left(\pi \rightarrow \pi^{*}, \varepsilon_{2550_{1}}-2+2 \mathrm{M}^{-1}\right): \mathbb{R}$ ( $\mathrm{KBr}$, stretching frequency $\mathrm{cm}^{-1}$ ): $338+(\mathrm{OH}), 1257$ (glycosidic aryl alkyl C-O-C asymmetrical). 1062 (glycosidic aryl alkyl C-O-C strmmetrical). $1365(\mathrm{C}=\mathrm{C}) .2923(\mathrm{CH}): \mathrm{MS}(\mathrm{m} / \mathrm{z})+19[\mathrm{M}+1]^{-}$. $2 \mathrm{D}-\mathrm{HSQCT}$ (DMSO- $\left.d_{b}\right)$ C1-sorbitol. ${ }^{1} \mathrm{H}$ NMR $\delta_{\text {ppo }}(500.13)$ Sor: 3.26 (H-1): Baluchiol: 1.87 (H-11). 1.48 (H-14): ${ }^{13} \mathrm{C} \mathrm{NMR} \delta_{\mathrm{ppm}}(125 \mathrm{MHz})$ : Sor $60.5(\mathrm{Cl}) .74 .4(\mathrm{C} 2) .71 .0(\mathrm{C} 3), 73.3(\mathrm{C} 4), 72.4(\mathrm{C} 5)$ : Bakuchiol: 26.7 (Cl1), 22.2 (Cl5), 14.0 (Cl7).

6-O-(6-D-Mannitol)baluchiol: Solid, UV $\left(\hat{t}_{\text {max }}\right): 191.5 \mathrm{~nm}$ $\left(\sigma \rightarrow \sigma^{*}, \varepsilon_{19.5}-9000 \mathrm{M}^{\mathrm{j}}\right) .199 .5 \mathrm{~nm}\left(\sigma \rightarrow \sigma^{*}, \varepsilon_{199.5}-6557 \mathrm{M}^{1}\right)$. $209.0 \mathrm{Im}\left(\sigma \rightarrow \sigma^{*} . \varepsilon_{209}-6486 \mathrm{M}^{-1}\right) .223 .0 \mathrm{~nm}\left(\sigma \rightarrow \pi^{*} . \varepsilon_{2330}\right.$ $-1+38 \mathrm{M}^{-1}$ ). $269.5 \mathrm{~nm}\left(\pi \rightarrow \pi^{*} . \varepsilon_{2635}-333 \mathrm{M}^{\mathrm{l}}\right)$ ): IR (KBr. stretching frequency $\left.\mathrm{cm}^{-1}\right): 3360(\mathrm{OH}), 1261$ (glycosidic aryll alkyl C-O-C asymmetrical). 1076 (glycosidic aryl alkyl C-O-C symmetrical). $1377(\mathrm{C}=\mathrm{C}) .2937(\mathrm{CH}): \mathrm{MS}(\mathrm{m} / \mathrm{z}) 419[\mathrm{M}+\mathrm{l}]^{-}$. 2D-HSQCT (DMSO- $d_{6}$ ) C6-mannitol: ${ }^{1} \mathrm{H}$ NMR $\delta_{p \text { man }}(500.13)$ Mannitol: $3.6 \mathrm{l}(\mathrm{H}-6)$ : Bakuchiol: $0.84(\mathrm{H}-17) .5 .08(\mathrm{H}-18):{ }^{13} \mathrm{C}$ NMR $\delta_{\mathrm{ppm}}(125 \mathrm{MHz})$ : Mannitol: $72.9(\mathrm{C} 2), 70.4(\mathrm{C} 3) .70 .8$ (C4). 72.9 (C5). 65.1 (C6); Baluuchiol: 38.2 (C 10), 13.9 (C17).

\section{Results and Discussion}

Glycosylation of bakuchiol using conventional reflux method resulted in gly cosides of D-glucose. D-ribose and D-arabinose only (Table 1 ) with yields in the range $9 \sim 51.4 \%$. Reactions carried out in supercritical $\mathrm{CO}_{2}$ media resulted in glycosides with aldohexoses - D-glucose. D-galactose and D-mannose: ketohexose - D-fructose: pentoses - D-ribose and D-arabinose; sugar alcohol-D-sorbitol and D-mannitol. The yields of the glycosides formed under $\mathrm{SCCO}_{2}$ conditions ranging from 9 to $46.6 \%$ (Table 2). Since, the $\mathrm{SCCO}_{2}$ conditions are mild, they are used as ideal conditions for the formation of glycosides
Table 1. Conversion yields and proportions of bakuchiol glycosides prepared by the Reflus method ${ }^{d}$

\begin{tabular}{|c|c|c|}
\hline \multirow{2}{*}{ No. } & \multirow{2}{*}{ Glycosides } & $\begin{array}{c}\text { Amyloglucosidase } \\
\text { catalysis }\end{array}$ \\
\hline & & $\begin{array}{ll}\text { Product } & \text { Yield } \\
\% & \text { proportions) } \\
(\%)^{\prime \prime}\end{array}$ \\
\hline
\end{tabular}

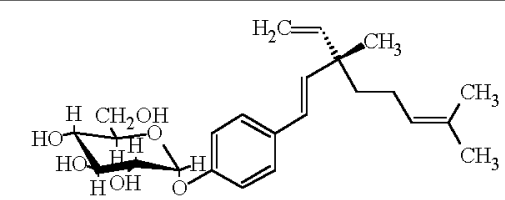

6-O-( $\alpha$-D-Glucopyranosyl)bakuchiol

$6-0-a(45)$

9.0

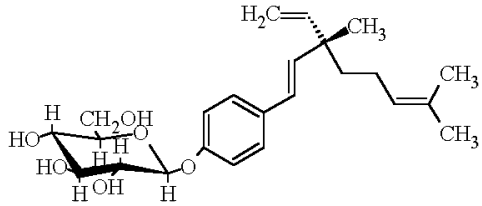

6-O-( $\beta-\alpha-\mathrm{D}-$ Glucopyranosyl ljbakuchiol $\quad 6-O-\beta$ (55)

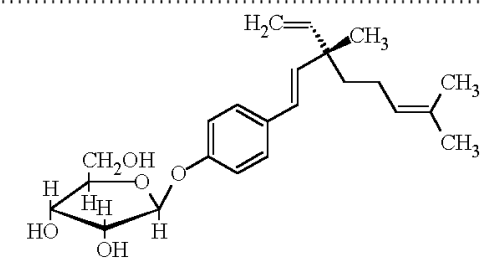

6-O-(a-D-Ribofuranosyl jbakuchiol $\quad 6-O-\alpha(23)$

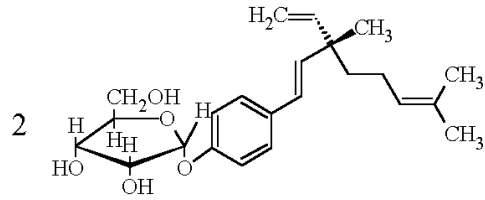

6-O-( $\beta$-D-Ribofuranosyl)bakuchiol

$6-O-\beta(53)$

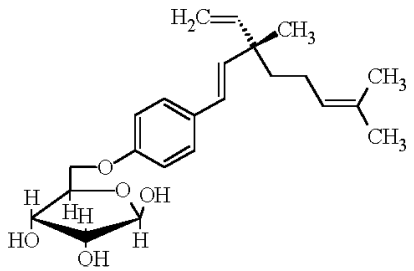

6-O-(5-D-Ribofuranosyl)bakuchiol $\quad 6-0-5(24)$

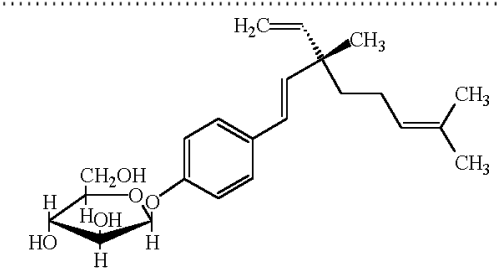

3 6-O-( $\alpha$-D-Arabinofuranosyl)bakuchiol $\quad 6-O-\alpha(27)$

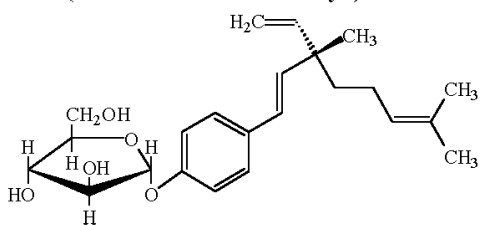

6-O-( $\beta$-D-Arabinofuranosyl)bakuchiol

$6-0-\beta(73)$

"Reaction refluxed at $68^{\circ} \mathrm{C}$ in di-isopropyl ether solvent at atmospheric pressure: "Bakuchiol $-0.5 \mathrm{mmol}$ and carbohydrate $1.0 \mathrm{mmol}$ : enzyme concentration $40^{\circ} .6$ w w carbolydrates: sol rent-di-isopropyl ether. DMF - $5.0 \mathrm{~mL} ; 0.1 \mathrm{mM}(1.0 \mathrm{~mL}) \mathrm{pH} 6.0$ phosphate buffer; incubation period

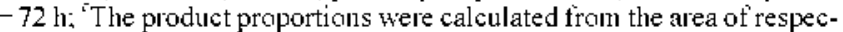
tive carbon signals. "Con ersion yields weere from HPLC with respect to the carbohydrate. Error in yield measurements is $=5^{\circ} \%$. 
Table 2. Conversion yields and proportions of bakuchiol glycosides prepared under $\mathrm{SCCO}_{2}$ condition ${ }^{2}$

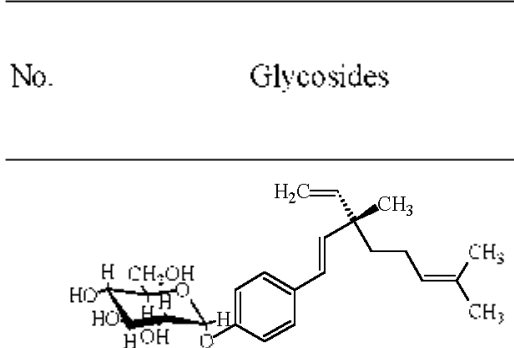

6-O-(a-D-Glucopyranosyl)bakuchiol

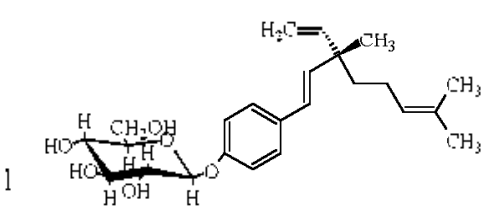

6-O-(B-D-Glucopyranosyl)bakuchiol

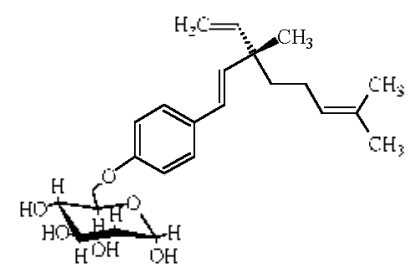

6-0-(5-D-Glucopyranos:])bakuchiol

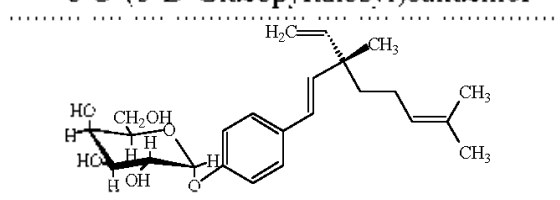

6-O-(a-D-Galactopyranosy])bakuchiol

2

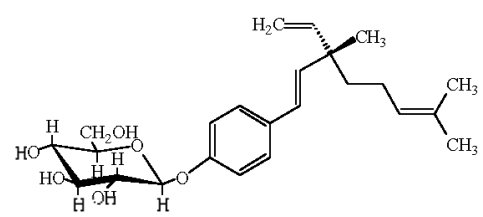

6-O-(B-D-Galactopyranosyl)bakuchiol

\begin{tabular}{c|c}
$\begin{array}{c}\text { Amyloglucosidase } \\
\text { catalysis }\end{array}$ & No. Glycosides \\
\hline Product Yield & No.
\end{tabular}

(\% proportions) $(\%)^{c}$
Amyloglucosidase catalysis $^{b}$

Product Yield (\% proportions) $(\%)^{d}$
$6-O-\beta(22)$

$6-0-\alpha(17) \quad 9$

6-0-6

arylated (61)

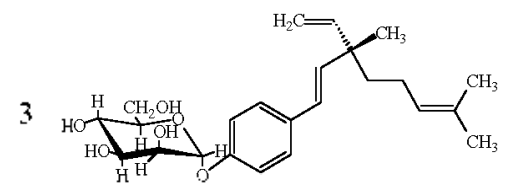

6-O-( $\alpha$-D-Mannoopyranosylibakuchiol $\quad$ 6-O- $\alpha$ 32.7

7

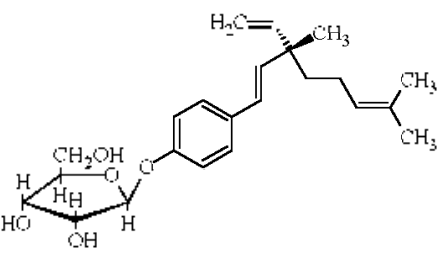

6-O-( $\alpha$-D-Ribofuranosyl libakuchiol

$6-0-\alpha(31)$

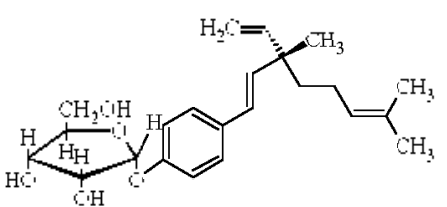

6-O-\{(B-D-Ribofuranosy] bakuchiol

$6-O-\beta(69)$

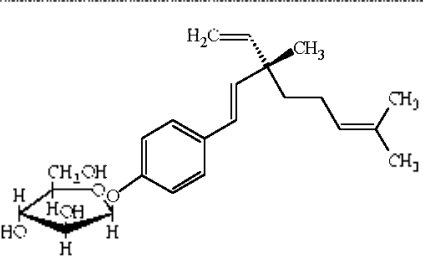

6-O-( $\alpha$-D-Arabinofuranosyl lbakuchiol $\quad 6-O-\alpha(38)$

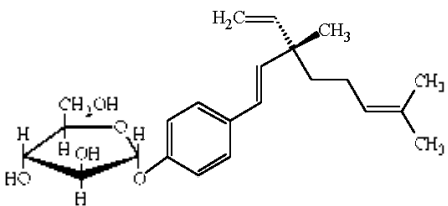

6-O-( $\beta-\mathrm{D}-$ Arabinofuranosyl jbakuchiol

$6-O-\beta(62)$

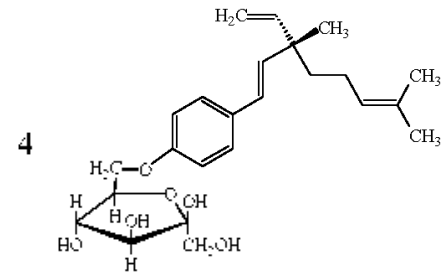

6-O-(6-D-Fructofuranosyl)bakuchiol

$6-0-6$

31.0

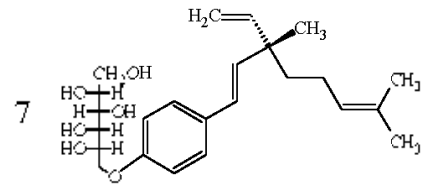

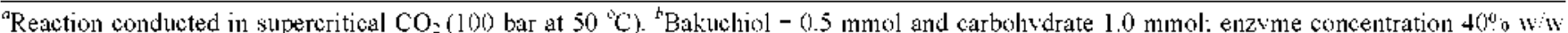
carbohydrates: solvent-DMF - $15.0 \mathrm{~mL} ; 0.1 \mathrm{mM}(1.0 \mathrm{~mL}) \mathrm{pH} 6.0$ phosphate buffer; incubation period - $24 \mathrm{~h}$. 'The product proportions were calculated from the area of respective carbon signals. "Confersion yields were from HPLC with respect to the carbolhydrate. Error in yield measurements is $\pm 5^{\circ} \mathrm{o}$. 
Table 3. IC $s_{0}$ values for Antioxidant activities of bakuchiol glycosides ${ }^{\pi}$

\begin{tabular}{lc}
\hline \multicolumn{1}{c}{ Compound } & IC $_{50}$ value (mM) \\
\hline Butylated Hydroxy Anisole & 0.029 \\
Bakuchiol & 1.24 \\
6-O-(D-Glucopyranosyl)bakuchiol & 1.34 \\
6-O-(D-Galactopyranosyl)bakuchiol & 1.28 \\
6-O-(D-Manuoopyranosyl)bakuchiol & 2.13 \\
6-O-(6-D-Fructofuranosyl)bakuchiol & 1.40 \\
6-O-(D-Ribofuranosyl)bakuchiol & 1.02 \\
6-O-(D-Arabinofuranosyl)bakuchiol & 1.20 \\
6-O-(1-D-Sorbitol)bakuchiol & 2.28 \\
6-O-(6-D-Mannitol)bakuchiol & 1.80 \\
\hline
\end{tabular}

"Antioxidant activity values deternined by DPPH radical scavenging method. Error in measurements is $\pm 5^{\circ} .0$.

with the above mentioned monosaccharides.

Sy nthesis of bakuchiol glycosides with carbohydrate molecules showed that except for D-ghicose. D-ribose and D-arabinose, the other carbohydrate molecules D-fructose. maltose. sucrose. lactose. D-sorbitol and D-mannitol did not undergo glycosylation under the conventional reflux conditions employed. This could be due to not-so-facile formation of the required oxocarbenium-ion intermediate ${ }^{19}$ with the other carbohydrate molecules at $68{ }^{\circ} \mathrm{C}$. Since the process conditions under $\mathrm{SCCO}_{2}$ media are mild. they served as ideal conditions for the formation of glycosides with many carbohydrates. Glycosylation resulted in enhancement of water solubility of bakuchiol.

Spectral characterization. Bakuchiol gly cosides were characterized by UV, IR, Mass, Optical rotation and 2DHSQCT NMR. UV spectra of bakuchiol glycosides showed shifts in the $\sigma \rightarrow \sigma^{*}$ band between $191.0 \mathrm{~nm}$ to $191.5 \mathrm{~nm} . \sigma \rightarrow \pi^{*}$ band at $199.5 \mathrm{~nm}$ to $231.5 \mathrm{~nm} . \pi \rightarrow \pi^{*}$ band at $259.5 \mathrm{~nm}$ to $275 \mathrm{~nm}$ and $\mathrm{n} \rightarrow \pi^{*}$ band at $288.5 \mathrm{rm}$. IR spectra showed shifts in the $\mathrm{OH}$ stretching frequency regain $2925 \mathrm{~cm}^{-1}-3397 \mathrm{~cm}^{-1}, \mathrm{C}=\mathrm{C}$ at 1347 $\mathrm{cm}^{-1} \sim 1440 \mathrm{~cm}^{-1}$. C-O-C astmmetrical at $1239 \mathrm{~cm}^{-1} \sim 1380 \mathrm{~cm}^{-1}$. C-O-C symmetrical stretching at $1049 \mathrm{~cm}^{-1} \sim 1085 \mathrm{~cm}^{-1}$ and $\mathrm{CH}$ at $2923 \mathrm{~cm}^{-1} \sim 2937 \mathrm{~cm}^{-1}$. 2DHSQCT NMR confirmed the formation of anomeric $\mathrm{Cl} \alpha$ and $\mathrm{Cl} \beta$ products as well as $\mathrm{C} 6$ arylated products. especially $\mathrm{Cl}$ and $\mathrm{C} 6$ arylated products of D-sorbitol and D-mannitol.

Antioxidant activity. Antioxidant activities of glycosides of bakuchiol and ACE inhibitory activities of bakuchiol glycosides are presented in Table 3 and t, respectively. Pure bakuchiol showed an antioxidant activity of $1.24 \mathrm{mM}$ ( $\mathrm{IC}_{50}$ value) as against $0.029 \mathrm{mM}$ for synthetic antioxidant BHA. Various glycosides of bakuchiol showed antioxidant activities ranging from 1.02 to $2.28 \mathrm{mM}$. Among the 8 glycosides prepared 6-O-(D-ribofuranosyl)bakuchiol and 6- $O$-(D-arabinofuranosyl) bakuchiol showed very low $I C_{\text {sa }}$ values of $1.02 \pm 0.102 \mathrm{mM}$ and $1.2 \pm 0.12 \mathrm{mM}$, while $6-0$-(D-galactopy ranosyl) bakuchiol ( 1.28 $\pm 0.128 \mathrm{mM}$ ) and $6-O$-(D-glucopy ranosyyl) bakuchiol ( $1.34 \pm$ $0.134 \mathrm{mM}$ ) showed significant $\mathrm{IC}_{\mathrm{Sij}}$ Values for antioxidant activity. Carbohydrate molecules themselves did not show antioxidant activities. Although phenolic $\mathrm{OH}$ group of bakuchiol is modified. it still showed marginally better antioxidant activity better than bakuchiol itself.

ACE inhibition. Bakuchiol glycosides were also tested for
Table 4. IC ${ }_{3 \mid 1}$ values for Angiotensin Converting Enzyme Inhibitory activities of bakuchiol glycosides"

\begin{tabular}{lc}
\hline \multicolumn{1}{c}{ Compound } & $\mathrm{IC}_{\xi(1}$ value $(\mathrm{mM})$ \\
\hline Enalapril & 0.071 \\
Bakuchiol & 0.74 \\
6-O-(D-Glucopyranosyl)bakuchiol & 1.33 \\
6-O-(D-Galactopyranosyl)bahuchiol & 1.22 \\
6-O-(D-Manuoopyranosyl)bakuchiol & 0.85 \\
6-O-(6-D-Fructofuranosyl)bakichchiol & 0.64 \\
6-O-(D-Ribofuranosy)bakuchiol & 0.85 \\
6-O-(D-Arabinofuranosyl)bakuchiol & 1.03 \\
6-O-(l-D-Sorbitol)bakuchiol & 1.20 \\
6-O-(6-D-Mannitol)bakuchiol & 0.89 \\
\hline
\end{tabular}

"Angiotensin Converting Enzyme Inhibitory activity determined by: Cushman and Cheung method, ${ }^{20}$ Error in measurements is $\pm 5^{\circ} .0$.

ACE inhibition. Bakuchiol gly cosides exhibited almost lesser $\mathrm{IC}_{5}$ values for ACE inhibition than bakuchiol itself. Among the different gly cosides prepared. $6-O$-(6-D-fructofuranosyl) bakuchiol. $0.64 \pm 0.06 \mathrm{mM} 6$ - $O$-(D-ribofuranosyl)bakuchiol. $0.85 \pm 0.09 \mathrm{mM}, 6-0$-(D-mannopyranosyl)bakuchiol. $0.85 \pm$ $0.09 \mathrm{mM}$ and 6-O-(6-D-mannitol)bakuchiol. $0.89 \pm 0.09 \mathrm{mM}$ exhibited better $\mathrm{IC}_{5, i}$ values than the other glycosides. $6-O-(\mathrm{D}$ Arabinofuranoșyl)bakuchiol, $1.03 \pm 0.10 \mathrm{mM}$ 6-O-(I-D-sorbitol) bakuchiol, $1.20 \pm 0.12 \mathrm{mM}, 6-O$-(D-galactopy ranosyl)bakuchiol. $1.22 \pm 0.12 \mathrm{mM}$ and $6-O$-(D-glucopyranosyl)bakuchiol, 1.33 $\pm 0.13 \mathrm{mM}$ showed high IC $\mathrm{C}_{50}$ Values for ACE inlibition. Bakuchiol and enalapril showed IC 5 ic values of $0.74 \pm 0.07 \mathrm{mM}$ and $0.071 \pm 0.007 \mathrm{mM}$ for $\mathrm{ACE}$ inhibition respectively $6-0-(6-\mathrm{D}-$ Fructofumnosyl) bakuchiol with $\mathrm{IC}_{5, j}$ value of $0.64 \pm 0.06 \mathrm{mM}$ has shown the best ACE inhibition than bakuchiol itself. Modification of the phenolic $\mathrm{OH}$ group by the carbohydrate molecule did not affect the ACE inhibition activity.

In contrast. under the supercritical $\mathrm{CO}_{2}$ atmosphere. glycosides with carbolydrate molecules of carbolydrates D-glucose, D-fnctose. D-ribose. D-sorbitol. D-arabinose. D-mannose and D-mannitol were formed and three disaccharides of maltose, sucrose and lactose were not detected. This could be due to the usefulness of the reaction medium which provided an ideal dielectric medium for the enzymatic reaction to occur with wide variety of carboly'drates. The yield of glycosides were in the range of $9-46.6 \%$.

Among the various carbohydrate molecules employed. particularly the glycosylation of aldo-hexoses like D-glucose. D-galactose and ketohexose D-fructose. aldo-pentoses like D-ribose. D-arabinose and sugar alcohol D-mannitol with phenolic $\mathrm{OH}$ group of bakuchiol converted bakuchiol into a freely water soluble compounds as well as enhance its biological activities also

\section{Conclusions}

Enzy'matic syntheses of water soluble Bakuchiol glycosides were reported first time. The reactions were carried in two different media: one by conventional reflux conditions and the other in supercritical $\mathrm{CO}_{2}$. Out of the eleven carbohydrate 
molecules employed for the reaction. D-glucose, D-ribose and D-arabinose gave glycosides in yields of $9.0 \%$ to $51.4 \%$ under conventional reflux method. Under supercritical $\mathrm{CO}_{3}$ conditions ( 100 bar pressure at $50^{\circ} \mathrm{C}$ ). bakuchiol formed glycosides with D-glucose. D-galactose. D-mannose. D-fructose. Dribose, $\mathrm{D}$-arabinose, $\mathrm{D}$-sorbitol and $\mathrm{D}$-mannitol inyield ranging from $9 \%$ to $46.6 \%$. Out of the bakuchiol glycosides prepared. 6-O-(6-D-fructofruranosyl)bakuchiol showed the best antioxidant (1.4 mM) and ACE inhibitory activities (0.6+ mM).

Aclnowledgments. Authors thank project assistants Mr. T. Ponrasu and Mr. R. E. Charles for the help during the experiment.

\section{References}

1. Kondo, Y.: Kato, A.: Kubota, Y.: Nozoe, S. Heterocycles 1990, 31, 187-190.

2. Latha, P. G.: Evans, D. A. Panikkar, K. R.; Tayawardhanan, K. K. Fitoterpia. 2000, 3, 223-231.

3. Katsura, H.: Tsutikiyama, R: Suzuki, A.: Kobayashi, M. Anti Microbial Agents Chemother 2001, $45(11), 3009-3013$.

4. Haraguchi, H.; Inoue, J.; Tamura, Y.; Mizutani, K. Phytotherapy' Research 2002, 16, 39-544.

5. Grice, H. C. Food and Chemical Toxicologv 1986, 2f, 1127-1 130.
6. Wichi. H. P. Food and Chemical Toxicology 1988, 26, 717- 723.

7. Tai, C. Y.; Huang, S. C.; Huang, M. S.; Liu, H. S. J. Chin. hist. Chem. Eng. 2001, 32(3), 269-275.

8. Russell, A. T.; Beckman, E. T. Enzyne Wicrob. Technol. 1991, $13(12), 1007$

9. Randolph. T. W; Blanch. H. W.; Pralusnitz, I. M.; Wilke, C. R. Biotechnol. Lett. 1985, 7(5), 325-328.

10. Mart,; A.; Chulalaksananukul, W.; Condoret, J. S.; Willemont, R. M; Durand, G. Biotechol. Lett. 1990, 12(1), 11-16.

11. Knez, M. H.; Krmelj, V. J. Supercrit. Flaids 1998, 14, 17-29.

12. Tumer, C.: Persson, M.: Mathiasson, L.: Adlercreutz, P.; King, J. W. Enzme Hicrob. Technol. 2001, 29, 111-121

13. Yu, H. M.; Lin, H. L; Wu, C. Y.; Tseng, M. T; Chen, S. T:; Jeyashoke, N.; Krisnangkura, K. J. Chin. Chem. Soc. 1999, $46(5)$, $647-650$

14. Chi, Y. M.; Nakamura, K; Yano, T. Agric. Biol. Chem. 1988, $52(6), 1541-1550$.

15. Nelson, L. A.: Foglia, T. A.: Marmer, W. N. J. Am. Oil Chem. Soc. 1996, 73(9), 1191-1195.

16. Miller, D. A.; Blanch, H. W.; Prausnitz, T. M. Ind. Eng. Chem. Res. 1991, 30, 939-946.

17. Compton, D. L.; King, J. W. J. Am. Oil Chent Soc. $2001,78(1), 43-47$.

18. Sumner, J. B.; Sisler, E. B. Arch. Biochem 1944, 4, 333-336.

19. Moon, J. H.; Tearo, J. J. Agri. Food Chent 1998, $48,5062-5065$.

20. Cushman, D. W.; Cheung, H. S. Biochent. Phamtacol. 1971, 20, $1637-1638$ 